\title{
The pattern of stress copying and interpretation of bodily sensation in patients with panic disorder
}

\author{
Jong-Chul Yang, MD, PhD \\ Department of Psychiatry, Chonbuk National University Medical School, Jeonju, Korea
}

Objectives: The purpose of this study was to investigate stress coping strategies and interpretation pattern of bodily sensation in patients with panic disorder.

Background: Patients with panic disorder have stressful life events in the months before the onset of the illness. And during panic attack, subjective intensity of fear was affected by interpretation pattern of bodily sensation. So we evaluated their characteristics.

Method: 101 panic patients and 60 normal controls were recruited. We evaluated the subjects by using Somato-Sensory Amplification Scale (SSAS), Symptom Interpretation Questionnaire (SIQ), Way of Stress Coping Questionnaire (SCQ), and Panic Disorder Severity Scale (PDSS). We analyzed the data using by independent t-test and Pearson correlation analysis $(p<0.05)$.

Result: Panic disorder patients had greater amplification of body sensation in SSAS $(28.10 \pm 7.94$ vs $22.63 \pm 7.28, p<0.05)$, significantly higher score in physical interpretation $(43.90 \pm 10.74$ vs $37.33 \pm 8.92, p<0.05)$, and lower score in environmental interpretation $(22.96 \pm 6.42$ vs $27.23 \pm 6.70, p<0.05)$ of SIQ than normal controls. PDSS scores were positively correlated with SSAS score and physical interpretation score of SIQ. In the SCQ rating of panic disorder patients, we observed significantly lower score in the emotional focused coping (16.21 3.48 vs $17.30 \pm$ $2.36, p<0.05)$.

Conclusion: Panic patients have greater amplification of body sensation, physical interpretation tendency on somatic symptoms and poor emotional focused stress copying strategy. These findings give a theoretical basis on necessity for interoceptive exposure, correction of maladaptive interpretation, and improvement of dysfunctional stress copying strategy for the treatment of panic disorder.

\section{References:}

1. These Clark,.DM, Salkovskis, PM, Ost LG, Breitholtz E, Koehler K, Westling BE, Jeavons A, \& Gelder M. Misinterpretation of body sensations in panic disorder. $J$ of consulting and clinical psychology 1997;65(2):203-213.

2. Casey LM, Oei TPS, Newcombe PA, Kenardy J. The role of catastrophic misinterpretation of bodily sensations and panic self-efficacy in predicting panic severity. Anxiety Dis 2004; 18:325-340.

Table 1. Sociodemographic characteristics of the subjects (Unit : Mean \pm SD)
\begin{tabular}{|lccc|}
\hline $\begin{array}{l}\text { Sociodemographic } \\
\text { characteristics }\end{array}$ & $\begin{array}{c}\text { Panic disorder patients } \\
\mathrm{N}=101(\%)\end{array}$ & $\begin{array}{c}\text { Normal controls } \\
\mathrm{N}=60(\%)\end{array}$ & $p$-value \\
\hline Age & $40.57 \pm 9.63$ & $42.95 \pm 11.11$ & NS \\
Sex & & & NS \\
Male & $55(54.46)$ & $32(53.33)$ & \\
Female & $46(45.54)$ & $28(46.67)$ & \\
Marital status & & & NS \\
Single & $15(14.85)$ & $10(16.67)$ & \\
Married & $86(85.15)$ & $50(83.33)$ & \\
Education & & & NS \\
$<7$ years & $6(5.94)$ & $4(6.67)$ & \\
$7-9$ years & $6(5.94)$ & $4(6.67)$ & \\
$10-12$ years & $37(36.63)$ & $19(31.66)$ & \\
$>12$ years & $52(51.49)$ & $33(55.00)$ \\
NS: not statistically significant by chi-square test \\
\hline
\end{tabular}

Table 2. Comparison of Somato-Sensory Amplification Scale and Symptom Interpretation Questionnaire between panic disorder patients and normal controls (Unit : Mean \pm SD)

\begin{tabular}{|lccc|}
\hline & $\begin{array}{c}\text { Panic disorder patients } \\
(\mathrm{N}=101)\end{array}$ & $\begin{array}{c}\text { Normal controls } \\
(\mathrm{N}=60)\end{array}$ & $p$-value \\
\hline $\begin{array}{l}\text { Somato-Sensoy } \\
\text { Amplification }\end{array}$ & $28.10 \pm 7.94$ & $22.63 \pm 7.28$ & $<0.001$ \\
Symptom Interpretation & & & \\
Physical Interpretation & $43.90 \pm 10.74$ & $37.33 \pm 8.92$ & $<0.001$ \\
Psychological Interpretation & $25.07 \pm 7.09$ & $25.23 \pm 6.08$ & 0.881 \\
Environmental Interpretation & $22.96 \pm 6.42$ & $27.23 \pm 6.70$ & $<0.001$ \\
\hline
\end{tabular}

Table 3. Comparison of coping strategy ratings for stress between panic disorder patients and normal controls (Unit : Mean \pm SD)

\begin{tabular}{|lccc|}
\hline & $\begin{array}{c}\text { Panic disorder patients } \\
(\mathrm{N}=101)\end{array}$ & $\begin{array}{c}\text { Normal controls } \\
(\mathrm{N}=60)\end{array}$ & $p$-value \\
\hline Active coping & $36.87 \pm 8.00$ & $38.37 \pm 5.43$ & 0.161 \\
Problem focused & $18.77 \pm 4.33$ & $19.77 \pm 3.48$ & 0.133 \\
Seeking social support & $18.10 \pm 4.70$ & $18.60 \pm 2.68$ & 0.390 \\
Passive coping & $36.94 \pm 5.65$ & $37.83 \pm 4.71$ & 0.305 \\
Emotion focused & $16.21 \pm 3.48$ & $17.30 \pm 2.36$ & 0.019 \\
Wishful thinking & $20.73 \pm 3.52$ & $20.53 \pm 2.84$ & 0.659 \\
\hline
\end{tabular}

Table 4. Correlation between PDSS and other factors

\begin{tabular}{|c|c|c|c|c|c|c|c|}
\hline & PDSS & $\begin{array}{l}\text { Active } \\
\text { coping }\end{array}$ & $\begin{array}{l}\text { Passive } \\
\text { coping }\end{array}$ & SSAS & $\begin{array}{l}\text { Physical } \\
\text { Interpre } \\
\text {-tation }\end{array}$ & $\begin{array}{l}\text { Psychological } \\
\text { Interpretation }\end{array}$ & $\begin{array}{l}\text { Environ- } \\
\text { mental } \\
\text { Interpre- } \\
\text { tation }\end{array}$ \\
\hline PDSS & 1 & & & & & & \\
\hline Active coping & 0.120 & 1 & & & & & \\
\hline $\begin{array}{l}\text { Passive } \\
\text { coping }\end{array}$ & 0.124 & $0.519+$ & 1 & & & & \\
\hline SSAS & $0.455^{+}$ & 0.075 & 0.197 & 1 & & & \\
\hline $\begin{array}{l}\text { Physical } \\
\text { Interpretation }\end{array}$ & $0.223^{*}$ & 0.115 & $0.253^{*}$ & $0.339 *$ & 1 & & \\
\hline $\begin{array}{l}\text { Psychological } \\
\text { Interpretation }\end{array}$ & 0.008 & $\overline{0.231 *}$ & 0.002 & $0.248 *$ & 0.141 & 1 & \\
\hline $\begin{array}{l}\text { Environmenta } \\
\text { Interpretation }\end{array}$ & 0.075 & -0.110 & 0.010 & 0.040 & $0.304 \uparrow$ & $0.272+$ & 1 \\
\hline $\begin{array}{l}\text { PDSS: Panic Disor } \\
\text { SSAS: Somato } \\
\text { *: significant at } p \\
+: \text { significant at }\end{array}$ & $\begin{array}{l}\text { der Severit } \\
\text { ensory Amp } \\
<0.05 \text { by } p \\
<0.01 \text { by p }\end{array}$ & $\begin{array}{l}\text { ty Scale } \\
\text { lification } \\
\text { pearson co } \\
\text { pearson co }\end{array}$ & $\begin{array}{l}\text { Scale } \\
\text { rrelation te } \\
\text { rrelation te }\end{array}$ & & & & \\
\hline
\end{tabular}

